\title{
Application of a Multidisciplinary Dysphagia and Nutrition Management System to Improve Safe Eating in Hospitalized Geriatric Patients
}

\author{
Guiyan Wen $^{1,2}$, Huixia Yu ${ }^{1,2, ~ *, ~ Q i u g u i ~ H u a n g ~}{ }^{1,2}$, Xiulan Deng ${ }^{1,2}$, Dongxiu Liang ${ }^{1,2}$ \\ ${ }^{1}$ Department of Gastroenterology, the First Affiliated Hospital of Jinan University, Guangzhou, China \\ ${ }^{2}$ School of Nursing, Jinan University, Guangzhou, China
}

Email address:

wengy78@126.com (Guiyan Wen), 18988970135@189.cn (Huixia Yu)

${ }^{*}$ Corresponding author

\section{To cite this article:}

Guiyan Wen, Huixia Yu, Qiugui Huang, Xiulan Deng, Dongxiu Liang. Application of a Multidisciplinary Dysphagia and Nutrition Management System to Improve Safe Eating in Hospitalized Geriatric Patients. American Journal of Nursing Science.

Vol. 10, No. 1, 2021, pp. 8-12. doi: 10.11648/j.ajns.20211001.12

Received: December 2, 2020; Accepted: December 14, 2020; Published: January 12, 2021

\begin{abstract}
This study aimed to explore the value of a multidisciplinary dysphagia and nutrition management system for the improvement of safe eating in hospitalized geriatric patients. We assembled a multidisciplinary team, consisting of a geriatric care team, a medical team, a rehabilitation team, professionals from the imaging center, and dietitians. Using the dysphagia and nutrition management system, we screened and assessed swallowing and eating dysfunction in geriatric patients admitted to our inpatient department and then provided instructions and swallowing exercises for patients with dysphagia. We measured the detection rate of patients with dysphagia and incidence of aspiration pneumonia. By applying the dysphagia and nutrition management system developed by our multidisciplinary team, the detection rate of patients with dysphagia increased significantly. Interventions of patients with dysphagia significantly decreased the incidence of aspiration pneumonia $(\mathrm{P}<0.05)$ and improved their nutritional status. Our multidisciplinary dysphagia and nutrition management system increased the detection rate of patients with dysphagia, improved safe eating, Prevention of aspiration and effective reduction of aspiration pneumonia in elderly patients. At present, the application of multidisciplinary cooperative swallowing and eating nursing system in the feeding safety of hospitalized elderly patients is relatively few, hoping to play a certain reference or guiding role in the clinical work of nursing peers.
\end{abstract}

Keywords: Aspiration Pneumonia, Dysphagia and Nutrition Management, Multidisciplinary Collaboration, Safe Eating

\section{Introduction}

Swallowing disorder management aims to improve safe eating. The goal of nursing care is to assess the swallowing and eating function of patients. Consequently, physicians can determine patients' nutritional status and take appropriate measures to improve patients' ability to eat independently, promote safe eating, and reduce inadvertent aspiration [1]. With the aging population, pharynx, esophagus site degenerative changes, Combined with the effects of diseases leading to dysphagia (including nervous system diseases, craniocerebral trauma, degenerative diseases, systemic diseases, tumors, infectious diseases, mental diseases and other diseases [2]), drugs and other factors, $32.5 \%$ of the elderly had dysphagia [3], The 2016 European Society for Dysphagia-European Association of Geriatrics White Paper reported [4]: the incidence of dysphagia in elderly people living alone is $30 \sim 40 \%$ [5], Elderly emergency patients have a $44 \%$ [6] rate, For the elderly / medical institutions, the incidence of the elderly is $60 \%$ [7]. Because of impaired swallowing, Ineffective swallowing of food and fluids, increased risk of aspiration [8], Many older people can not eat safely and efficiently, Occurrence of aspiration leading to aspiration pneumonia, asphyxia and other serious complications, It directly causes the increase of medical cost, the extension of hospitalization time and the increase of care pressure. At present, there is a consensus among domestic experts on the evaluation and treatment of dysphagia [9-10], 
but the recognition rate of dysphagia in elderly patients is low, And the clinical screening, treatment and nursing reports of multidisciplinary cooperation are less. In July 2018, the nursing department in our hospital assembled a multidisciplinary team to assess swallowing function in geriatric patients and provide more competent care for patients with dysphagia. The multidisciplinary system achieved good outcomes, reported below.

\section{Materials and Methods}

\subsection{Baseline Information}

We recruited a total of 31,994 geriatric patients (over the age of 65 years) who were admitted to our hospital from June 2017 to July 2019 and randomly assigned them to two groups. The control group consisted of 15,877 patients who were hospitalized from June 2017 to June 2018; they were evaluated using routine screening questions for symptoms of dysphagia and then given regular dietary care. The experimental group had a total of 16,117 patients who were hospitalized between July 2018 and July 2019; they were screened, evaluated, and treated according to the multidisciplinary dysphagia and nutrition management system. The detection rate of patients with dysphagia and the incidence of aspiration pneumonia during hospitalization were measured in the two groups. The body mass index (BMI), serum albumin $(\mathrm{Alb})$, and hemoglobin $(\mathrm{Hb})$ levels of patients with dysphagia in the two groups were also recorded.

\subsection{Methods}

\subsubsection{Control Group}

i. Routine screening questions for symptoms of dysphagia and nutrition management

Geriatric patients were evaluated using routine screening questions on symptoms of dysphagia within eight hours of admission, and those with symptoms, such as choking and swallowing deficits, were considered to have dysphagia. Patients diagnosed with dysphagia were given food through the oral route or nasogastric tube according to the severity of their symptoms.

\subsubsection{Experimental Group}

i. Assembly of the multidisciplinary team for the development of a dysphagia and nutrition management system

A multidisciplinary team for the management of swallowing and feeding was assembled, and the responsibilities of the members were outlined. The members of the team included a geriatric care nurse, a primary nurse, a physician, a rehabilitation therapist, a dietitian, and an imaging technician. The responsibilities of the members were as follows.

The primary nurse conducts preliminary screening of dysphagia in the admitted geriatric patient and reports the results to the physician and geriatric care nurse. Based on their feedback, the primary nurse carries out swallowing and feeding interventions.

The geriatric care nurse is responsible for further assessment of the patient and instructs the primary nurse on providing individualized intervention for patients with moderate or severe dysphagia. The geriatric care nurse also conducts training sessions on the management of patients with dysphagia, and is responsible for the quality control of the care.

The physician makes decisions on the following: the feeding route of the patient based on the patient's diagnosis, treatment they receive, results of the evaluation of swallowing deficits, and recommendations from the geriatric care nurse.

The rehabilitation therapist is responsible for further assessment and rehabilitation intervention of patients with severe dysphagia.

The dietitian is responsible for the nutritional guidance for malnourished patients.

The imaging technician collaborates with the rehabilitation therapist to conduct video fluoroscopic swallowing study (VFSS) on patients with severe dysphagia to determine the incidence of aspiration when the patient swallows.

ii. Development of guidelines for dysphagia and nutrition management

Guidelines for the early screening of dysphagia and nutrition management for geriatric patients were developed with reference to the recommendations from the 2017 edition of the Chinese Expert Consensus on the Evaluation and Treatment of Dysphagia [9]. The specific steps were as follows.

a. Screening and Evaluation of Swallowing Deficits

Preliminary screening is conducted. Within eight hours of admission, the primary nurse asks the patient if they had symptoms of dysphagia and then conducts the Repetitive Saliva Swallowing Test and the Water-Swallowing Test (WST). WST, first proposed by Japanese researchers in 1982, classifies swallowing function into five levels. Level I indicates that the patient has a normal swallowing function and can swallow water in one gulp without choking or coughing (under 5 seconds). Level II indicates mild swallowing deficits, where the patient can swallow water in two or more gulps without choking or coughing. Level III indicates moderate dysphagia, where the patient can swallow water in one gulp but with coughing or choking. Level IV indicates severe dysphagia, where the patient can swallow water in two or more gulps with coughing or choking. Level $\mathrm{V}$ indicates profound dysphagia, where the patient shows frequent coughing or choking. Patients evaluated to be level I or II are considered negative for aspiration, whereas patients at levels III-V are considered aspiration-positive. The test is non-invasive and easy to conduct in clinical practice.

After preliminary screening, patients evaluated at levels III-V in the WST (i.e. diagnosed with dysphagia) are subjected to further assessment of swallowing function by the geriatric care nurse using the volume-viscosity swallow test (V-VST). The test includes three levels of volumes, namely, small $(5 \mathrm{ml})$, medium $(10 \mathrm{ml})$, and large $(20 \mathrm{ml})$, and three levels of viscosity, namely, low (watery), medium (thick 
paste), and high (jelly-like). Using the different combinations, a complete test requires a total of nine gulps. The patient's swallowing process is observed, and based on the observation, the nurse determines whether there is risk associated with eating according to the indicators of safety and effectiveness.

VFSS is the gold standard for the diagnosis of dysphagia. During V-VST, the rehabilitation therapist and the imaging technician perform the VFSS to determine visually whether there is a high risk of aspiration, or the presence of silent and overt aspiration during the patient's swallowing process. VFSS also provides a reference for the determination of a patient's feeding route, compensatory strategies, and swallowing exercises.

b. Swallowing Exercises

For patients evaluated at levels III-V in the WST who are determined to be stable after the consultation between the nurse and the physician, swallowing exercise regimens are created after consultation with the rehabilitation department. For patients diagnosed with dysphagia, cold stimulation training [11], transcutaneous electrical nerve stimulation, and swallowing training are used to improve the sensory function of the oral cavity and swallowing function, thereby increasing the safety of swallowing [12]. Patients are re-evaluated weekly for swallowing function and detailed records are kept.

\section{c. Safe Eating Management}

The geriatric care nurse, physician, and rehabilitation therapist discuss and determine each patient's feeding route, permitted food consistency, and eating technique, including the amount per bite and speed of eating.

Formulation and implementation of the management standards for oral feeding

Before eating: A good eating environment should be created. The nurse should assist the patient in preparing for the meal (e.g., wash the face and hands, clean the oral cavity, set up the table). The nurse should clear the room of irrelevant personnel and avoid loud talking to give the patient a comfortable, quiet, and relaxed environment.

Body position for eating: The patient should try to sit up and keep the torso straight at 90 degrees during eating; patients with weak physical strength can lie on their healthy side at 30-60 degrees in a semi-recumbent position, with the neck and head bent slightly forward. The shoulder on the patient's hemiplegic side should be supported by a cushion. The person who feeds the patient should stand at the healthy side of the patient. This body position can reduce the risk of aspiration caused by food regurgitation to the nasal cavity. When the muscles in front of the neck are relaxed, the larynx can be lifted easily, thereby reducing the risk of aspiration.

Food selection and preparation: Patients are fed strictly in accordance with the diet plan established after assessment. In addition to choosing food that would make up a balanced diet, ensuring safety is also a major concern; the food chosen should be easy to swallow. Patients evaluated as level I in the WST are given normal food and boneless meals. Patients who have been evaluated at level II in the WST are given eating instruction and basic swallowing training by the nurse, and the food given should be pureed, semi-liquidized, or minced. Patients at levels III-V who passed the V-VST should have a pureed diet (barium swallow test is recommended to rule out silent aspiration). For a pureed diet, a blender should be used to mix and crush cooked foods (e.g., cereals, meat, vegetables, beans) to make them easy to transport and swallow. For drinking liquids, thickeners should be added to increase the viscosity. Moreover, the consistency should be adjusted according to the safety and effectiveness of swallowing demonstrated in V-VST. Patients evaluated at levels III-V in the WST who failed the V-VST are not allowed to eat through the mouth and should be put on nasogastric or nasojejunal tube, or undergo gastrostomy for enteral nutrition (according to the standard of care).

When eating: The cutlery should be a spoon with a thick edge and a long handle with a volume of less than $5 \mathrm{ml}$. This will make it easier to control the amount of food per spoon. The amount per spoon should start at $1-4 \mathrm{ml}$, and then increased to a mouthful suitable for the patient. The patient should eat slowly and take small bites. For those who have a slow swallowing reflex or have residue after swallowing, they can swallow in several gulps and clear their throats frequently, and they should be carefully observed for any symptoms of swallowing difficulties when eating.

After eating: patients should remain in the sitting or semi-recumbent position for at least half an hour. Their oral cavity should be cleaned to reduce the growth of bacteria in the mouth and prevent silent aspiration.

Placement of nasogastric tube, nasojejunal tube, and gastrostomy for enteral nutrition

A standard of care for enteral nutrition is established and implemented.

Evaluation of quality of care

The nurse should continue to reassess the patient's swallowing function at least once per week. They should be evaluated for improvements in swallowing function, occurrence of aspiration, and occurrence of aspiration pneumonia.

Dysphagia and nutrition management index

A dysphagia and nutrition management index is established. Quality control should include the screening rate of swallowing function, detection rate of dysphagia, and rate of satisfactory care for dysphagia and nutrition management. Four aspects are evaluated, namely, the accuracy of a nurse's screening test for dysphagia, nutrition management of geriatric patients with dysphagia, implementation of measures, and nursing records. Monthly quality checks are conducted by the head of the nursing team, the geriatric care nurse, and the head nurse, respectively, and the results are summarized and analyzed for continuous quality improvement.

\subsubsection{Statistical Methods}

SPSS 16.0 was used to analyze the data, and frequency and rate were used for statistical description. The comparison between the two groups was performed by $\chi 2$ test for discrete data. We used $t$-test for independent samples. $\mathrm{P}<0.05$ indicated statistical significance. 


\section{Results}

\subsection{Comparison of Baseline Characteristics}

Table 1. Comparison of the baseline characteristics of the two groups of patients.

\begin{tabular}{lllll}
\hline \multirow{2}{*}{ Group } & Sex (cases) & & Age & \multicolumn{2}{c}{ Average duration of illness } \\
\cline { 2 - 5 } & male & female & years, $(\mathbf{X} \pm \mathbf{S})$ & $\mathbf{d},(\mathbf{X} \pm \mathbf{S})$ \\
\hline Control group $(\mathrm{n}=15,877)$ & 9,065 & 6,792 & $65.49 \pm 13.74$ & $11.19 \pm 6.47$ \\
Experimental group $(\mathrm{n}=16,117)$ & 9,477 & 6,640 & $66.52 \pm 12.82$ & $10.89 \pm 5.17$ \\
$\mathrm{t} /{ }^{\mathrm{x} 2}$ value & 0.759 & & 1.352 & 0.960 \\
P-value & 0.764 & & 0.456 & 0.370 \\
\hline
\end{tabular}

\subsection{Comparison of Detection Rate of Dysphagia}

Table 2. Comparison of the detection rate of dysphagia between the two groups.

\begin{tabular}{lll}
\hline Group & Number of cases of dysphagia detected & Detection rate of dysphagia (\%) \\
\hline Control group $(\mathrm{n}=15,877)$ & 677 & $4.27 \%$ \\
Experimental group $(\mathrm{n}=16,117)$ & 2168 & $13.45 \%$ \\
\hline
\end{tabular}

\subsection{Comparison of Incidence of Aspiration Pneumonia Among Patients with Dysphagia}

Table 3. Comparison of the incidence of aspiration pneumonia among patients with dysphagia in the two groups.

\begin{tabular}{lll}
\hline Group & Number of cases of aspiration pneumonia & Incidence of aspiration pneumonia (\%) \\
\hline Control group $(\mathrm{n}=677)$ & 111 & $16.49 \%$ \\
Experimental group $(\mathrm{n}=2,168)$ & 69 & $3.18 \%$ \\
\hline
\end{tabular}

The detection rate of dysphagia in the control group was $4.27 \%$ (677 out of 15,877), and among these patients with dysphagia, aspiration pneumonia had an incidence rate of $16.49 \%$ (111 cases). In comparison, the detection rate of dysphagia in the experimental group was $13.45 \%$ (2,168 out of 16,117), and we determined 69 cases of aspiration pneumonia in patients with dysphagia (incidence rate: $3.18 \%$ ). The detection rate of dysphagia and incidence of aspiration pneumonia were significantly different between the control and experimental groups $(\mathrm{P}<0.05)$.

The experimental group had the following average values for BMI, serum Alb level, and Hb level: $25.41 \pm 3.24,35.52 \pm$ $2.53 \mathrm{~g} / \mathrm{L}$, and $113.87 \pm 12.24 \mathrm{~g} / \mathrm{L}$, respectively. The levels of serum $\mathrm{Alb}$ and $\mathrm{Hb}$ of the two groups were significantly different.

\section{Discussion}

Dysphagia is a common clinical symptom in multiple disciplines and is the primary risk factor for aspiration. Its occurrence is positively correlated with age [13]. Early detection and intervention of dysphagia can significantly reduce the risk of complications, such as aspiration and malnutrition [14]. To prevent aspiration and ensure safe eating in geriatric patients, medical professionals need to detect dysphagia accurately, and the assessment of swallowing function is the most critical first step. Nurses play an extremely important role in the detection and diagnosis of dysphagia in patients. The Chinese Expert Consensus on the Evaluation and Treatment of Dysphagia (2017 edition) [3] specifically recommends multidisciplinary collaboration for the assessment and treatment of patients with dysphagia. The multidisciplinary dysphagia and nutrition management system developed in the present study was highly effective for increasing the detection rate of dysphagia in hospitalized patients. The assessment of patients in the experimental group was more refined compared with routine screening. We created a guideline and flowchart that covered the process from assessment to treatment, making the system more specific and feasible.

Different dysphagia and nutrition management strategies should be adopted for patients with different levels of dysphagia severity. In our study, we revised the dysphagia and nutrition management process for patients at high risk of aspiration outlined in the Chinese Expert Consensus on Evaluation and Treatment of Dysphagia (2017 edition) based on our clinical nursing experience. We focused on the development of different nursing care plans for different severity levels of dysphagia and achieved good results. Dysphagia has a considerable effect on the nutritional status and physical condition of geriatric patients, and dysphagia and malnutrition are mutually causal. Therefore, a multi-level management system with individualized nutrition plans for patients with dysphagia can significantly improve patient prognosis and reduce the incidence of complications. However, the multidisciplinary cooperation mode of dysphagia management is still being explored, and the communication and coordination mechanism needs to be further improved [15].

\section{Conclusion}

The establishment of a multidisciplinary cooperative swallowing and eating nursing system, such as medical 
treatment, nursing, rehabilitation, nutrition and imaging, to carry out swallowing function screening, swallowing function training and safe eating care for patients with high risk of aspiration, which is conducive to early identification of patients with dysphagia, implementation of safe eating nursing intervention, effective prevention of aspiration, prevention or correction of malnutrition, reduction of aspiration pneumonia, and ensuring nursing safety.

\section{References}

[1] Chen, C. and Peng, G., Standards for Clinical Nursing Documentation (Specialist Edition). City, State: Guangdong Science and Technology Press, 2009, 1: 100.

[2] Dou Zulin. assessment and treatment of dysphagia. Version 2. Beijing: people's Health Publishing House.

[3] Crary, M., Sura, L., Madhavan, A., et al. (Year). Dysphagia in the elderly: management and nutritional considerations. Clinical Interventions in Aging: 287.

[4] Baijens LW, Clave P, Cras P, et al. European society for swallowing disorders-european union geriatric medicine society white paper: oropharyngeal dysphagia as a geriatric syndrome. Clin Interv Aging, 2016, 11: 1403-1428.

[5] Lin LC, Wu SC, Chen HS, et al. Prevalence of impaired swallowing in institutionalized older people in Taiwan. J Am Geriatr Soc, 2002, 50 (6): 1118-1123.

[6] Ja L. Evaluation and treatment of swallowing disorders. Austin, TX: College-Hill Press, 1983: 1245-1255.

[7] Clave P, Rofes L, Carrion S, et al. Pathophysiology, relevance and natural history of oropharyngeal dysphagia among older people. Nestle Nutr Inst Workshop Ser, 2012, 72: 57-66.
[8] Chinese expert consensus on Family Nutrition Management in elderly patients with dysphagia (2018 Edition). Chinese Journal of Evidence-based Medicine, June 2018, vol. 18, No. 6.

[9] Chinese expert consensus group on rehabilitation assessment and treatment of dysphagia. Chinese Expert Consensus on Assessment and Treatment of Dysphagia (2017 Edition) J].] Evaluation Chinese Journal of Physical Medicine and Rehabilitation, 2017, 39 (12): 881-892.

[10] Chinese expert consensus group on rehabilitation assessment and treatment of dysphagia. Chinese Expert Consensus on Assessment and Treatment of Dysphagia (2017 Edition) treatment and rehabilitation management [J]. articles Chinese Journal of Physical Medicine and Rehabilitation, 2018, 40 (1): $1-9$.

[11] Sciortino, K. F., Liss, J. M., Case, J. L., et al. (2003). Effects of mechanical, cold, gustatory, and combined stimulation to the human anterior faucial pillars. Dysphagia, 18 (1): 16-26.

[12] Bhatt, A. D., Goodwin, N., Cash, E., et al. (2015). Impact of transcutaneous neuromuscular electrical stimulation on dysphagia in patients with head and neck cancer treated with definitive chemoradiation. Head and Neck Journal for the Sciences and Specialties of the Head and Neck, 37 (7): 10511056.

[13] Xia, W., Bai, J., Xia, L., et al. (2009). Investigation on the status quo of incidence of aspiration in hospitalized patients. Chinese Nursing Research, 23 (31): 2848-2849.

[14] Chang, H., Zhao, J., Zhang, S., et al. (2018). Evaluation of the effect of food consistency on the decrease of aspiration in patients with dysphagia after stroke. Chinese Journal of Nursing, 1: 32-35.

[15] Huang Shiju Cai Youdi Li Xiaoling and others. Evaluation of screening and grading intervention in patients with dysphagia in high risk departments led by nurses [J] Chinese Journal of Nursing, November 2018, vol. 53, No. 11, 1303-1308. 